\title{
Is controlled hypotension or stroke volume variation the main determinant of surgical bleeding in functional endoscopic sinus surgery? A pilot study
}

\author{
Di Mauro R. ${ }^{1}$, Dauri M. ${ }^{2}$, Di Girolamo S. ${ }^{1}$, Silvi M.B. ${ }^{2}$, Boezi M. ${ }^{3}$, Lucci F. $^{2}$ \\ 1University Tor Vergata, Rome, Otorhinolaryngology, Rome, Italy, \\ 2University Tor Vergata, Rome, Anaesthesiology \& Intensive Care, Rome, Italy, \\ 3San Pietro Fatebenefratelli, Anaesthesiology \& Intensive Care, Rome, Italy
}

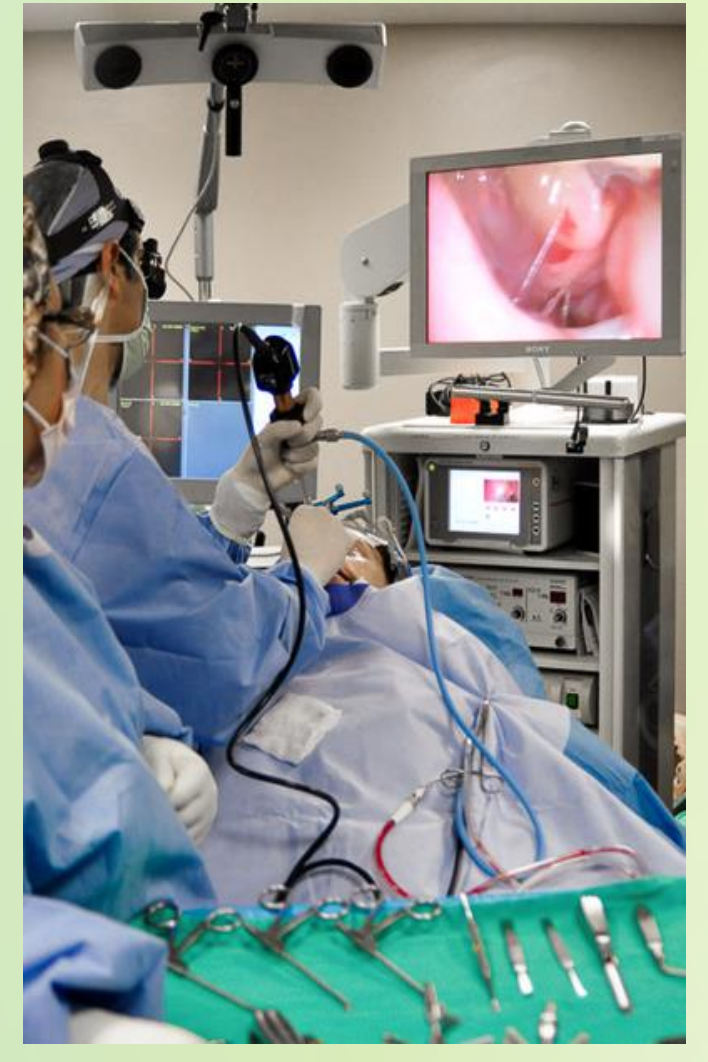

Background and Goal of the Study FESS is a minimally-invasive surgical technique for patients with nasal and paranasal sinus pathology that has become popular worldwide. Surgical bleeding reduces operative field visibility, increases the incidence of serious vascular, orbital, and intracranial complications and prolongs surgical duration. Anaesthetic technique can reduce these complications.

Epinephrine injection into the nasal mucosa, head elevation and controlled hypotension can be used to minimise bleeding. Hypotension carries risks and in sometimes a MAP of $60 \mathrm{mmHg}$ does not reduce surgical bleeding. Study goal was to see if there is a correlation between bleeding and haemodynamic parameters using a new non-invasive monitor, the CLEARSIGHT (Edwards Lifesciences).

\section{Materials and Methods}

We enrolled 35 patients undergoing FESS into a prospective study. We had approval from the ethics committee and procedures performed were in accordance with the 1964 Helsinki declaration. Inclusion criteria: ASA 1-3, age 18-80.

Exclusion criteria: Neoplastic lesions and clotting disorders. Anaesthesia induction: Propofol $2-3 \mathrm{mg} / \mathrm{kg}$, sufentanil 0.2-0.4 $\mathrm{mcg} / \mathrm{kg}$ and rocuronium $0.6 \mathrm{mg} / \mathrm{kg}$.

Maintenance: Propofol/remifentanil TCI.

Monitoring: Standard ASA plus CLEARSIGHT to assess stroke volume index (SVI) and stroke volume variation (SVV). Haemodynamic targets: MAP $60 \mathrm{mmHg}$, SVV >12\% and SVI $40 \mathrm{ml} / \mathrm{m} 2 /$ beat.

In order to ensure consistency as much as possible, all procedures were performed by one surgeon and following the same surgical steps. The surgical operation was divided in 10 times (from T0 to T9) corresponding to a specific surgical phase. Intraoperative bleeding was assessed using the Fromm scale (from 0 , no bleeding to 5 , worst bleeding). The surgeon was blinded to the monitor.

\begin{tabular}{|l|l|l|l|l|l|l|l|}
\hline & Cl & SVI & SVV & PR & SYS & DIA & MAP \\
\hline P value & & & & & & & \\
& 0.17 & 0.22 & 0.04 & 0.85 & 0.74 & 0.84 & 0.89 \\
\hline
\end{tabular}

\section{Results and Discussion}

An adequate surgical field was achieved in 30/35 patients $(\mathrm{p}<0,01)$. An adequate surgical field correlated with $\mathrm{SVV}>12 \%\left(\mathrm{R}^{\wedge} 2=0,39\right)$, better than hypotension $(\mathrm{MAP}=60 \mathrm{mmHg})\left(\mathrm{R}^{\wedge} 2=0,022\right)$. These haemodynamic targets were well tolerated in all cases.
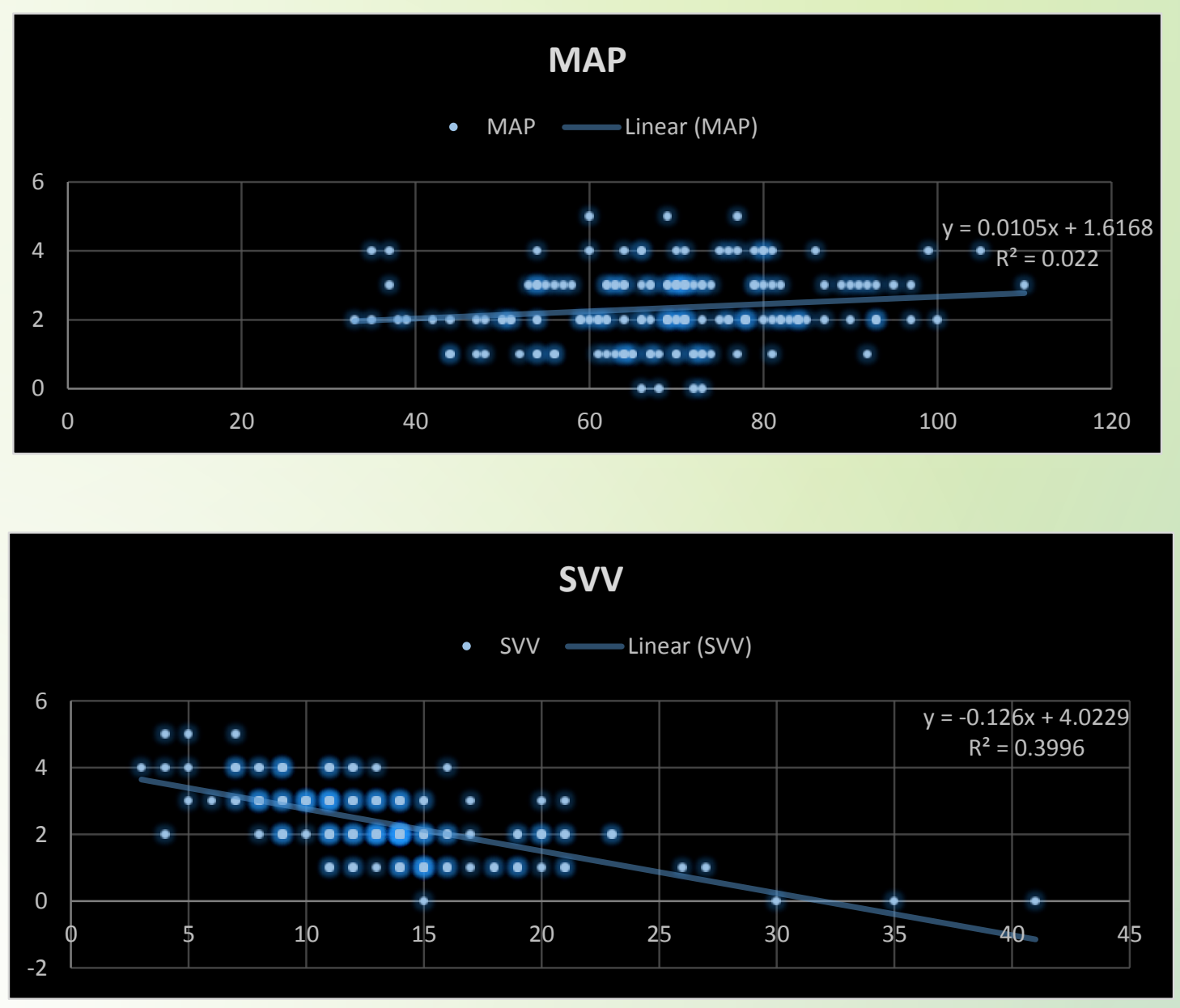

\section{Conclusion}

The results showed a strong negative correlation between surgical bleeding and volemic status as determined by a SVV > 12\%. Targeting higher SVV achieved shorter surgical duration and minimal risk of complications. Further studies are required.
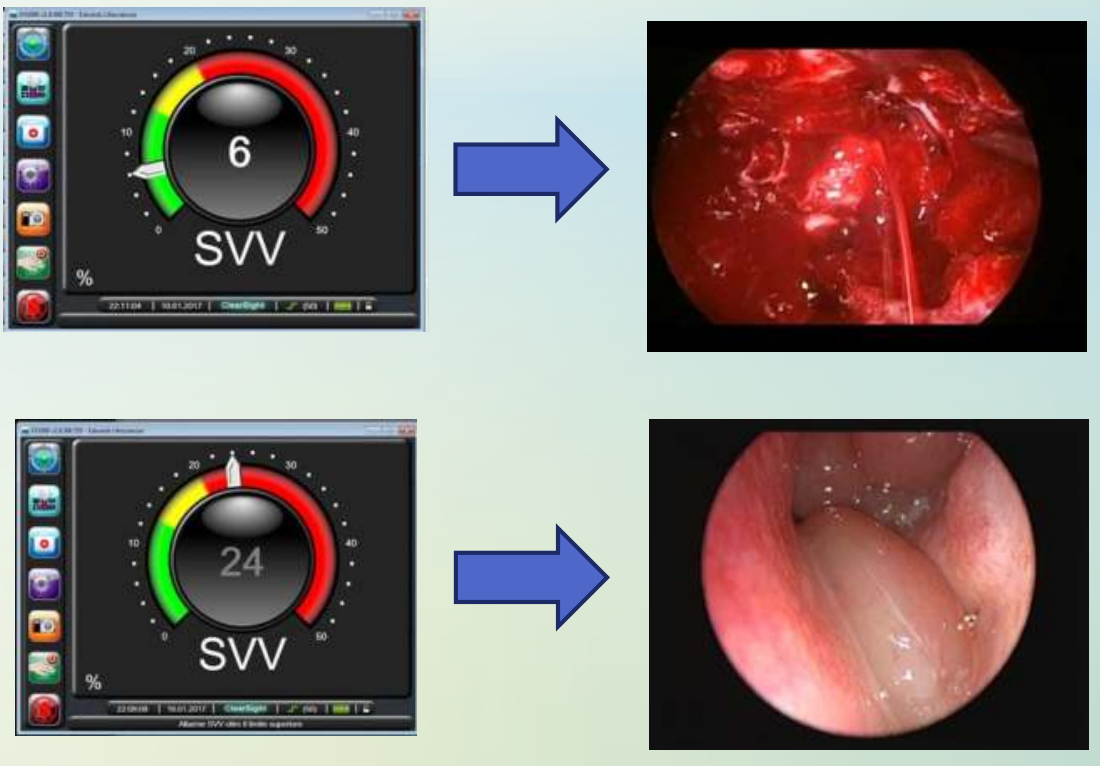

\section{References}

